\title{
Editorial
}

\section{Managing obstetric haemorrhage: is it time for a more personalised approach?}

\author{
A. Shah ${ }^{1,2}$ and R. E. Collis ${ }^{3}$ \\ 1 Specialist Registrar, Nuffield Department of Anaesthesia, John Radcliffe Hospital, Oxford, UK \\ 2 NIHR Doctoral Research Fellow, Radcliffe Department of Medicine, University of Oxford, UK \\ 3 Consultant Anaesthetist, Department of Anaesthetics, Cardiff and Vale University Health Board, Cardiff, UK
}

Correspondence to: A. Shah

Email: akshayshah@doctors.org.uk

Accepted: 12 March 2019

Keywords: coagulation; Postpartum haemorrhage treatment; transfusion

Twitter: @DocAShah; @Rachel42863647

The incidence of maternal mortality and morbidity secondary to major obstetric haemorrhage continues to rise globally, although only a small proportion of this increase can be explained by changes in risk factors such as maternal age, pre-eclampsia, abnormally invasive placentation and operative deliveries [1]. In this issue of Anaesthesia, McNamara et al. [2] provide 4 years of follow-up data on maternal and transfusion-related outcomes following the introduction of a rotational thromboelastometry $\left(\right.$ ROTEM $^{\circledR}$, Tem International GmBH, Munich, Germany)-guided algorithm to treat major obstetric haemorrhage [3]. The authors observed a statistically significant reduction in the volume of blood products transfused, along with improved maternal outcomes, when compared with the time period before the introduction of the algorithm during which the use of formulaic 'shock packs' consisting of fixed ratios of blood products was standard practice. This is an important study that raises a number of key issues around the continued use of formulaic transfusion strategies, choice of fibrinogen replacement therapy and use of viscoelastic haemostatic assays in major obstetric haemorrhage. Importantly, the study demonstrates that genuine improvements in major obstetric haemorrhage rates and outcomes are possible.

\section{Massive transfusion: one size does not fit all}

Guidelines and reviews currently give inconsistent messages on the use of formulaic infusion of fresh frozen plasma in fixed ratios with red blood cells in major obstetric haemorrhage. Although new guidance still recommends the use of formulaic infusions in patients at high-risk of bleeding (e.g. placental abruption) or after the first four units of red blood cells have been given and coagulation results are not available, some still recommend the use of fixed ratios from the outset $[4,5]$. These recommendations were extrapolated from improvements seen in trauma and military medicine [6], but there are no data to support their use in obstetric haemorrhage.

Although guidelines recommend using prothrombin time (PT) and activated partial thromboplastin time (APTT) to guide therapy, it is well recognised that they are poor predictors of bleeding [7]. During maternal haemorrhage, PT and APTT can remain within the normal range even until blood loss reaches $4000-5000 \mathrm{ml}$ [8]. In addition, these tests have a turnaround time of 60-90 min making the results less relevant in an acutely changing situation and the blind use of blood products common practice.

Of greater importance perhaps is that the procoagulant profile of the parturient at term, where the normal Clauss fibrinogen is 4-6 g.l $\mathrm{I}^{-1}$, is very different from a non-pregnant adult involved in a major road traffic accident [9]. Although trauma-induced coagulopathy is associated with early and profound fibrinogen depletion almost universally, it is becoming increasingly clear that the coagulopathy associated with obstetric haemorrhage differs significantly depending on its underlying cause $[9,10]$. The most 
common causes of major obstetric haemorrhage such as uterine atony and genital tract trauma are usually associated with no significant coagulopathy and fibrinogen levels are relatively well-maintained even with large blood losses. With current guidance, these patients may receive large and unnecessary volumes of blood products $[8,10]$. In contrast, placental abruption may be associated with early coagulopathy even before bleeding is apparent, while abnormally invasive placentas (e.g. accreta) are associated with very rapid haemorrhage leading to hypofibrinogenaemia and thrombocytopenia [10]. These differences are described in the study by McNamara et al.

\section{Fibrinogen replacement}

Fibrinogen is the most important biomarker in predicting progression to severe obstetric haemorrhage [11, 12]. It falls progressively as blood loss increases while APPT and PT remain normal until blood loss is greater than $4000 \mathrm{ml}$ [12].

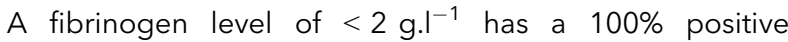
predictive value for progression from moderate to severe haemorrhage and current guidelines recommend maintaining a fibrinogen level above this during major obstetric haemorrhage [4]. Two therapeutic options are available to replace fibrinogen - cryoprecipitate and fibrinogen concentrate [13]. Cryoprecipitate is the product of choice to treat acquired hypofibrinogenaemia in the UK, North America and Australia. It has, however, been largely withdrawn from use in many European countries as it is produced from multiple donors and therefore carries a higher risk of viral transmission. The fibrinogen concentrations in cryoprecipitate also varies from 3 to $30 \mathrm{~g} . \mathrm{I}^{-1}$ [14], leading to a potentially inconsistent effect, and requires two to three freeze-thaw cycles before administration, which can cause a delay in correction of coagulopathy.

For these reasons, many European countries have opted for fibrinogen concentrate as the main product for replacing fibrinogen. Advantages include: an increased viral safety profile; standardised concentration of fibrinogen; near-patient storage at room temperature in powder form allowing immediate access and administration and no blood incompatibility issues [14]. Fibrinogen concentrate is, however, more expensive and a recent economic evaluation showed that even after cryoprecipitate wastage, fibrinogen concentrate is at least twice as expensive [15].

McNamara et al. demonstrated a reduction in the use of blood products, notably fresh frozen plasma, and similar findings have been observed in other studies in nonobstetric studies using fibrinogen concentrate [16].
Although these results appear to be promising, a systematic review comparing the efficacy of fibrinogen concentrate vs. cryoprecipitate found very little high-quality data to draw meaningful conclusions [17]. The results of two large randomised controlled trials comparing cryoprecipitate vs. fibrinogen in trauma [NCT02745041] and cardiac surgery [NCT03037424] are awaited but there is no adequately powered study in obstetric major haemorrhage to compare the clinical efficacy of the two formulations, their safety profile including thrombotic complications, and wider cost effectiveness issues.

\section{Viscoelastic haemostatic assays for obstetric haemorrhage}

Although the use of viscoelastic haemostatic assays is supported by evidence in cardiac surgery [18], their use on labour ward has been debated for nearly 15 years [19]. The National Institute for Health and Care Excellence (NICE) guidance does not recommend their use during obstetric haemorrhage except in the context of clinical trials [20]. The two commonest assays are thromoboelastography (TEG ${ }^{\circledR}$, Haemonetics Corp, Baintree, MA, USA) and ROTEM and the manufacturers have added an assay to measure fibrinogen (functional-fibrinogen TEG and FIBTEM-ROTEM). The main advantages of these assays are the quick turnaround time, with an evaluation of all stages of clot kinetics available in 5$10 \mathrm{~min}$, especially with the new automated devices such as TEG-6 and ROTEM Sigma, which can also be performed by non-laboratory personnel [21].

There have been a number of published algorithms, including the work from McNamara et al., which have demonstrated the successful use of ROTEM parameters to safely guide blood product administration. There is less evidence supporting the use of TEG, although pragmatically there does not seem to be a reason why similar algorithms will not be available in time. The correlation between Clauss fibrinogen and FIBTEM has an $r^{2}$ value of about 0.6 , which demonstrates that they do not measure the same thing, but both have been found to be equally good at predicting progression to major obstetric haemorrhage [22]. There is less evidence around viscoelastic haemostatic assay cut-off values for the use of fresh frozen plasma where an EXTEM CT time of 75-100 s has been advocated [3, 4, 23]. The poor prediction of PT and APTT for bleeding probably shows that this is a less important part of the algorithm, especially in obstetric practice, where generalised clotting abnormalities, which can be identified by significant derangements in the ROTEM-EXTEM and TEG parameters, are a rare and late feature of major obstetric haemorrhage. This was demonstrated in the observational arm of a study by Collins 
et al. which enrolled 605 women experiencing moderate to severe postpartum haemorrhage [24]. Fresh frozen plasma was not administered if FIBTEM A5 $>15 \mathrm{~mm}$ or the bleeding had stopped. Ninety-eight per cent of patients did not receive fresh frozen plasma despite a median blood loss of $1500 \mathrm{ml}$ and none developed coagulopathy as defined by PT or APTT > 1.5 times the midpoint of the normal range.

Based on recent publications, the most recent British Society of Haematology guidelines on the use of viscoelastic haemostatic assays now differ from previous NICE guidance [25]. By evaluating recent data from obstetric haemorrhage $[3,24]$, they now recommend that fibrinogen replacement may improve haemostasis if FIBTEM A5 is $<7 \mathrm{~mm}$, or $<12 \mathrm{~mm}$ with ongoing bleeding (Grade 2C). Of equal importance, they recommend that fibrinogen replacement is unlikely to improve clinical haemostasis if FIBTEM A5 is > $12 \mathrm{~mm}$ (Grade 2B). The current study by McNamara et al. adds to the knowledge in this field.

\section{When should viscoelastic haemostatic assays be used during obstetric haemorrhage?}

Two important factors that require consideration are the anticipated frequency of use of viscoelastic assays and economic costs.

In the current study by McNamara et al., ROTEM was performed when blood loss was estimated at $1500 \mathrm{ml}$ in around $2.7 \%$ of the maternity population, although the authors did not state how many ROTEM tests were actually performed in total. They also had a significant number of severe abruptions with coagulopathy, and although the paper does not state the exact time a ROTEM was performed, it is likely that an early ROTEM test was used to guide early fibrinogen therapy in this high-risk sub-group. It is especially interesting that the incidence of a low fibrinogen (FIBTEM $<12 \mathrm{~mm}$ ) was present in less than $1 \%$ of their population and only half were given fibrinogen concentrate. In the Obstetric Bleeding Strategy for Wales (OBS Cymru) protocol [23], ROTEM is performed when there is measured blood loss $>1000 \mathrm{ml}$ with ongoing concern (around $5 \%$ of the population) or in the high-risk abruption group before bleeding has become a problem.

At one of the institutions participating in the Collins et al. study [24], there were significant reductions in red blood cell and plasma usage, obstetric haemorrhages with blood loss $>2500 \mathrm{ml}$, and critical care admissions across the whole population during the time of the study. When this study ended in November 2015, there was an increase in large haemorrhages similar to before the study and a local inquiry identified themes such as: the multidisciplinary team not attending the mother's bed-side quickly; a return to estimating blood loss instead of the more accurate gravimetric assessment; viscoelastic haemostatic assays not being performed early and delays in obstetric and haemostatic interventions [23]. After these factors were rectified and guidance both on appropriate escalation during major obstetric haemorrhage and using ROTEMdriven blood products within a specific pathway, outcomes improved to those found during the study by Collins et al. [24].

With only $0.3 \%$ of the maternal population in the study by McNamara et al. receiving fibrinogen concentrate, their major improvements in major obstetric haemorrhage outcomes are likely to be multifactorial, and this strongly suggests that early knowledge of haemostatic competence can influence management. If viscoelastic haemostatic assay results are normal, focus can shift towards obstetric management and not blood products. If viscoelastic haemostatic assay results are abnormal, this automatically identifies the woman as high risk, alerts the team to causes such as placental abruption and facilitates more rapid and targeted treatment of blood components. Although there is concern that the majority of labour wards may not use the technology frequently enough to allow staff to gain experience or to make it cost effective, the effect on team behaviour and a more coordinated approach to $\mathrm{PPH}$ management may improve outcomes and provide value for money even in the smaller units. Currently, unpublished results from OBS Cymru [26] have shown improved major obstetric haemorrhage outcomes in maternity units of all sizes, although cost effectiveness is still unknown.

\section{Future directions}

Pragmatic randomised controlled trials are needed to establish the role of viscoelastic haemostatic assays in major obstetric haemorrhage, combined with robust cost effectiveness analyses. The use of fibrinogen concentrate rather than cryoprecipitate would be an important part of this analysis. Process evaluations and mixed-method approaches, embedded within randomised studies, can be used to further explore how early knowledge of coagulation results, when compared with standard laboratory testing, may influence patient outcomes. If such studies and cost analyses are not performed, there is a danger that a two-tier maternity service will develop where smaller units will be not able to justify the costs of adopting the algorithms illustrated by McNamara et al. and others. Currently, this study shows excellent results and certainly adds to the 
literature in major obstetric haemorrhage, but because it was a single-centre observational study, it is unlikely to influence national guidance such as NICE.

\section{Acknowledgements}

AS is a Trainee Fellow of Anaesthesia and is being supported by an NIHR Doctoral Research Fellowship (DRF2017-10-094). RC has received research support and honoraria from TEM International, Werfen and CSL Behring.

\section{References}

1. Kramer MS, Berg C, Abenhaim H, et al. Incidence, risk factors, and temporal trends in severe postpartum hemorrhage. American Journal of Obstetrics and Gynecology 2013; 209 : e1-7.

2. McNamara $H$, Kenyon $C$, Smith $R$, Mallaiah S, Barclay P. Four year's experience of a ROTEM ${ }^{\circledR}$ guided algorithm for treament of coagulopathy in obstetric haemorrhage. Anaesthesia 2019; 74: ???-???

3. Mallaiah S, Barclay P, Harrod I, Chevannes C, Bhalla A Introduction of an algorithm for ROTEM-guided fibrinogen concentrate administration in major obstetric haemorrhage. Anaesthesia 2015; 70: 166-75.

4. Mavrides E, Allard S, Chandraharan E, et al. Prevention and management of postpartum haemorrhage. British Journal of Obstetrics and Gynaecology 2016; 124: e106-49.

5. Hunt BJ, Allard S, Keeling D, et al. A practical guidelines for the haematological management of major haemorrhage. British Journal of Haematology 2015; 170: 788-803.

6. Penn-Barwell JG, Roberts SA, Midwinter MJ, Bishop JR. Improved survival in UK combat casualties from Iraq and Afghanistan: 2003-2012. Journal of Trauma and Acute Care Surgery 2015; 78: 1014-20.

7. Shah A, Stanworth SJ, McKechnie S. Evidence and triggers for the transfusion of blood and blood products. Anaesthesia 2015; 70(Suppl 1): e3-5.

8. Collins PW, Lilley G, Bruynseels D, et al. Fibrin-based clot formation as an early and rapid biomarker for progression of postpartum hemorrhage: a prospective study. Blood 2014; 124: 1727-36.

9. Collis RE, Collins PW. Haemostatic management of obstetric haemorrhage. Anaesthesia 2015; 70(Suppl 1): 78-86.

10. Green L, Knight M, Seeney F, et al. The haematological features and transfusion management of women who required massive transfusion for major obstetric haemorrhage in the UK: a population based study. British Journal of Haematology 2016; 172: 616-24.

11. Charbit B, Mandelbrot L, Samain E, et al. The decrease of fibrinogen is an early predictor of the severity of postpartum hemorrhage. Journal of Thrombosis and Haemostasis 2007; 5 : 266-73.
12. de Lloyd L, Bovington R, Kaye A, et al. Standard haemostatic tests following major obstetric haemorrhage. International Journal of Obstetric Anesthesia 2011; 11: 135-41.

13. Collins PW, Solomon C, Sutor K, et al. Theoretical modelling of fibrinogen supplementation with therapeutic plasma, cryoprecipitate, or fibrinogen concentrate. British Journal of Anaesthesia 2014; 113: 585-95.

14. Levy JH, Goodnough LT. How I use fibrinogen replacement therapy in acquired bleeding. Blood 2015; 125: 1387-93.

15. Okerberg CK, Williams LA 3rd, et al. Cryoprecipitate AHF vs. fibrinogen concentrates for fibrinogen replacement in acquired bleeding patients - an economic evaluation. Vox Sanguinis 2016; 111: 292-8.

16. Morrison GA, Koch J, Royds M, et al. Fibrinogen concentrate vs. fresh frozen plasma for the management of coagulopathy during thoraco-abdominal aortic aneurysm surgery: a pilot randomised controlled trial. Anaesthesia 2019; 74: 180-9.

17. Jensen $\mathrm{NH}$, Stensballe J, Afshari A. Comparing efficacy and safety of fibrinogen concentrate to cryoprecipitate in bleeding patients: a systematic review. Acta Anaesthesiologica Scandinavica 2016; 60: 1033-42.

18. Wikkelso A, Wetterslev J, Moller AM, Afshari A. Thromboelastography (TEG) or rotational thromboelastometry (ROTEM) to monitor haemostatic treatment in bleeding patients: a systematic review with meta-analysis and trial sequential analysis. Anaesthesia 2017; 72: 519-31.

19. Hunt BJ, Lyons G. Thromboelastography should be available in every labour ward. International Journal of Obstetric Anesthesia 2005; 14: 324-5.

20. Whiting $P, A l M$, Westwood $M$, et al. Viscoelastic point-of-care testing to assist with the diagnosis, management and monitoring of haemostasis: a systematic review and cost-effectiveness analysis. Health Technolology Assessment 2015; 19: 1-228.

21. Schenk B, Gorlinger K, Treml B, et al. A comparison of the new ROTEM ${ }^{\circledR}$ sigma with its predecessor, the ROTEMdelta. Anaesthesia 2019; 74: 348-56

22. Huissoud C, Carrabin N, Audibert F, et al. Bedside assessment of fibrinogen level in postpartum haemorrhage by thromboelastometry. British Journal of Obstetrics and Gynaecology 2009; 116: 1097-102.

23. Collins PW, Bell SF, de Lloyd L, Collis RE. Management of postpartum haemorrhage: from research into practice, a narrative review of the literature and the Cardiff experience. International Journal of Obstetric Anesthesia 2019; 37: 106-17.

24. Collins PW, Cannings-John R, Bruynseels D, et al. Viscoelastometry guided fresh frozen plasma infusion for postpartum haemorrhage: an observational study: OBS2. British Journal of Anaesthesia 2017; 119: 422-34.

25. Curry NS, Davenport R, Pavord $S$, et al. The use of viscoelastic haemostatic assays in the management of major bleeding: a British Society for Haematology Guideline. British Journal of Haematology 2018; 182: 789-806.

26. NHS Wales Informatics Service. Obstetric bleeding strategy for wales. ???. http://www.1000livesplus.wales.nhs.uk/obs-cymru (accessed 11/03/2019). 